IMECE2009-11213

\title{
NATURAL CONVECTION FROM ISOTHERMAL HORIZONTAL CYLINDERS
}

\author{
Ian M. O Gorman \\ University of Dublin, Trinity College \\ Dublin, Ireland \\ Gerard Byrne \\ University of Dublin, Trinity College \\ Dublin, Ireland
}

\author{
Prof. Darina B. Murray \\ University of Dublin, Trinity College \\ Dublin, Ireland \\ Dr. Tim Persoons \\ University of Dublin, Trinity College \\ Dublin, Ireland
}

\begin{abstract}
The research described here is concerned with natural convection from isothermal cylinders, with a particular focus on the interaction between a pair of vertically aligned cylinders. Prime attention was focused on how the local heat transfer characteristics of the upper cylinder are affected due to buoyancy induced fluid flow from the lower cylinder. Tests were performed using internally heated copper cylinders with an outside diameter $30 \mathrm{~mm}$ and a vertical separation distance between the cylinders ranging from two to three cylinder diameters. Plume interaction between the heated cylinders was investigated within a Rayleigh number range of $2 \times 10^{6}$ to $6 \times 10^{6}$. Spectral analysis of the associated heat transfer interaction is presented showing that interaction between the cylinders causes oscillation of the thermal plume. The effect of this oscillation is considered as a possible enhancement mechanism of the heat transfer performance of the upper cylinder.
\end{abstract}

\section{INTRODUCTION}

Tubular heat exchangers are one of the most fundamental, simple and widely used mechanisms for transferring heat between two fluids. This basic, yet fundamental device is extensively used in a wide range of both industrial and domestic applications however there still remain fundamental questions to be answered concerning the interaction between vertically aligned horizontal cylinders which make up the main component of any heat exchanger. The current research is aimed at investigating these fundamental questions in an effort to optimize design concerning tubular heated cylinders in terms of the associated buoyancy driven heat transfer.
Natural convection around a single horizontal cylinder has been studied by many previous researchers such as Morgan [1] and Churchill and Chu [2]. The work of these researchers has established a simple relationship between the mean Nusselt number $(\overline{N u})$ and the Rayleigh number (Ra) of a single cylinder and is given by the expressions:

$$
\overline{N u}=C R a^{n}
$$

$$
\overline{N u}=0.36+0.518\left(\frac{R a}{\left[1+(0.559 / \mathrm{Pr})^{9 / 16}\right]^{16 / 9}}\right)^{1 / 4}
$$

Where $\mathrm{C}$ and $\mathrm{n}$ represent constants based on the Rayleigh number range, $\operatorname{Pr}$ represents the Prandtl number of the surrounding fluid as calculated based on the film temperature. It is clear from the above relationships that essentially, a very simple relationship exists between the mean Nusselt number of a single cylinder and that of the Rayleigh number. However, when a second cylinder is introduced to the system vertically inline above this first cylinder the performance characteristics of this second cylinder greatly differ from that of a single cylinder due to interaction of the temperature and flow fields. The mean Nusselt number of the second cylinder is no longer 
only a function of the Rayleigh number but is also affected by the vertical spacing between the cylinders. The performance of the second cylinder can either be enhanced or reduced relative to that of a single cylinder due to plume interaction from the lower cylinder with the upper cylinder. Eckert and Soehngen [3] have investigated heat transfer from a vertical cylinder array and have found that the induced temperature and velocity fields due to the buoyant plume from downstream cylinders have opposite effects. For a pair of inline cylinders the heat transfer rate from the upper cylinder was found to decrease with decreasing cylinder spacing due to a decrease in the local temperature difference as a result of the buoyant plume, while at larger spacing an increase in the associated heat transfer was found to occur due to the increase in the local fluid velocity having a forced convection effect on the upper cylinder.

It has been found from previous researchers that when a single cylinder is constrained in either the horizontal or the vertical direction, the heat transfer is dramatically affected compared to that of a single cylinder in an infinite medium. Atmane et al. [4] investigated the heat transfer from a single cylinder vertically confined by a free water surface. It was found that vertical confinement can enhance heat transfer from a single cylinder. The primary effect of this confinement is to increase the heat flux on the upper part of the cylinder. This research also found that at small separation distances the heat transfer to the surrounding fluid is reduced. Above a separation distance of three cylinder diameters it was found that the vertical confinement had no effect on heat transfer. Studies carried out by Yaghoubi et al. [5] investigated the effect of an adiabatic ceiling on heat transfer within horizontal tubular arrays. The studies found that the ceiling height greatly affects the interaction between adjacent cylinders in an array. A Mach Zehnder interferometer was used to visualize the temperature variations within a tubular array and smoke particles to visualize the flow variations. These researchers found that decreasing the ceiling height caused the recirculation strength to increase on the upper part of the cylinder which also had the effect of causing the boundary layers on the neighbouring cylinders to combine and form a developing flow between the cylinders.

Much research has also been carried out into investigating the effect of horizontal confinement on heat transfer from tubular arrays. Tokura et al. [6] experimentally investigated the effect of parallel plates enclosing a vertical cylinder array. This research showed that confinement using parallel adiabatic plates, causes a chimney effect which results in an increase of the heat transfer from the array. The enhancement effect of cylinders set between plates increases by $10-15 \%$ from an array in open space when the cylinder spacing is large and the distance between the plates is about $2-$ 6D. G. Cesini et al. [7] investigated numerically the flow around a horizontally confined array. This research showed that the fluid motion is strongly dependant on the aspect ratio of the cavity, it was also found that there is no effect of the plates relating to heat transfer promotion when the distance between the parallel plates is three times the cylinder diameter.

Comparatively little research has been carried out into the free convection flow around a pair of vertically aligned cylinders relative to the volume of work carried out on the study of a single cylinder. The free convection flow structure within a horizontal cylinder array is complex and is influenced by several geometric and operating parameters. Included in these parameters are the Rayleigh number $(\mathrm{Ra})$, the Prandtl number (Pr), the vertical and horizontal spacing $(\mathrm{S} / \mathrm{D}, \mathrm{H} / \mathrm{D}$ where $\mathrm{D}$ is the cylinder diameter) the cylinder arrangement, as well as the effects of both horizontal and vertical confinement. Each of these parameters has an influence on the plume development as well as on the velocity and temperature distribution within the generated plume. Sparrow and Niethammer [8] explored the effect of separation distance over a Rayleigh number range of $2 \times 10^{4}-2 \times 10^{5}$. From the results of their research it was found that, at small separation distances degradation is generally the rule, while at larger separation distances enhancement predominates. An optimum separation distance to maximize heat transfer from the upper cylinder was found to occur at a separation distance of between 7 and 9 cylinder diameters. However these tests were limited to low Rayleigh numbers.

While the interactions between vertically aligned cylinders have been studied by previous researchers such as Marsters [1], Yousefi and Ashjaee [9], Sparrow and Niethammer [8] and Reymond et al. [10] using both experimental and analytical techniques, according to Tokura et al. [6] few papers have been published analysing the local Nusselt number variation with cylinder arrays. This report investigates the local Nusselt number variation of both a single cylinder and a pair of vertically inline cylinders at separation distances ranging from two to three cylinder diameters within a Rayleigh number range of $2 \times 10^{6}$ and $6 \times 10^{6}$. A frequency analysis of the plume oscillation within the cylinder array is presented and this oscillation considered as a possible mechanism for heat transfer enhancement from the upper cylinder.

\section{EXPERIMENTAL SETUP AND PROCEDURE}

An experimental rig was constructed as part of this research, the design of which would approximate testing of a tubular section of infinite length contained within an infinite fluid medium. As such, the test rig was designed to minimize both horizontal and vertical confinement effects and to minimize the impact of end effects which would affect the fluid flow around the cylinder. Research carried out by Tokura et al. [6] relating to horizontal confinement showed that there was no effect of parallel end plates on the heat transfer performance when the distance between the plates was greater than three times the cylinder diameter; the test rig was therefore designed with this fact in mind. Atmane et al. [4] showed that no vertical confinement occurred when the distance between the top of the cylinder and the fluid surface was greater than $3 \mathrm{D}$. So that 
results would not be affected by the fore mentioned confinement issues an experimental test rig was developed measuring $900 \mathrm{~mm}$ high, $900 \mathrm{~mm}$ long and $300 \mathrm{~mm}$ wide as indicated in figure 1 . This setup provided a cylinder length to diameter ratio of 10:1 which was required to mitigate end effects. Water was chosen as the working fluid for this analysis. Air was deemed unsuitable as a working fluid due to the very high temperatures that would be required in order to reach the required Rayleigh number range and also due to the difficulty of isolating a control volume of air that would not be influenced by the surroundings.

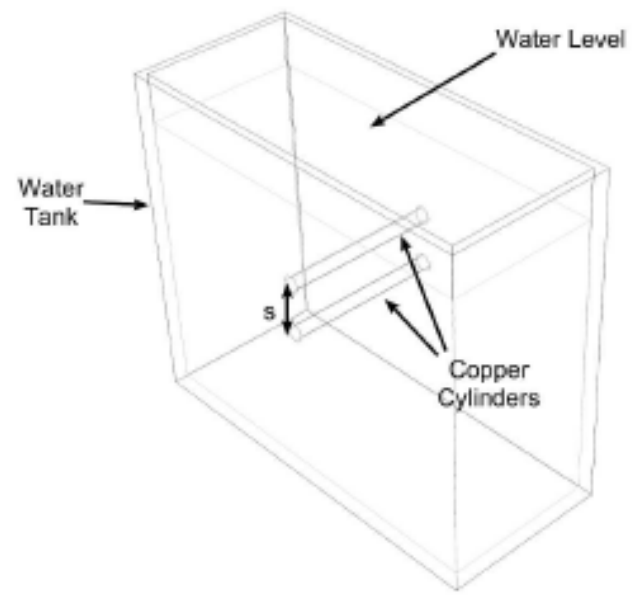

Figure 1. Schematic representation of experimental set-up.

This test rig consists of a bulk volume of approximately 200litres of deoxygenated water; this large volume of water is required in order to provide a large thermal mass to avoid bulk water temperature drift during testing. The tank is constructed primarily from Polymethylmethacrylate (Plexiglas), thus avoiding corrosion problems. This tank is restrained with an aluminium framing structure which provides structural rigidity for the tank.

Identical copper cylindrical test sections were manufactured, each containing two $500 \mathrm{~W}$ electric cartridge heaters. The diameter $(D=30 \mathrm{~mm})$ was chosen as it is a standard piping size used in many industrial heat exchangers and is also a common size piping used in many large scale heating systems. The lower cylinder of each spacing tested is positioned $300 \mathrm{~mm}$ from the base of the test rig. Each of these test sections is instrumented with a flush mounted heat flux sensor (RdF Micro-Foil $\left.{ }^{\circledR} \quad 27036-2-R d F\right)$ and an internally mounted T-type thermocouple. The instrumented cylinders are designed to be externally rotated about their axis of symmetry so that the surface distribution of heat flux can be measured around the circumference while not introducing a large disturbance to the surrounding fluid, as can be seen in figure 2 .

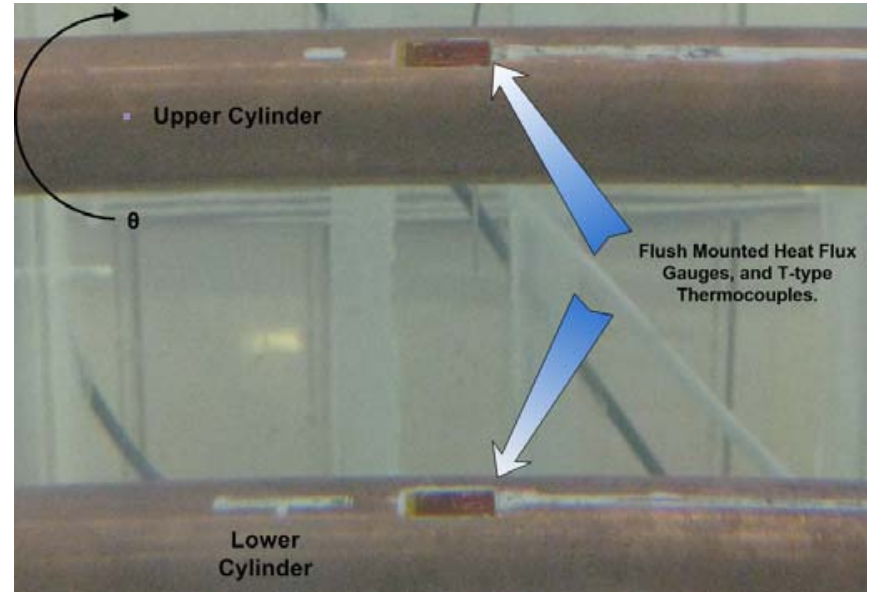

Figure 2. Instrumented Cylinders showing the location of the Flush mounted Heat Flux sensors and T-type Thermocouples.

To minimize radiation losses the surface of each of the test sections is highly polished to reduce the emissivity of the copper cylinders to approximately 0.1 , therefore heat transfer due to radiation is considered negligible. Conduction losses through the ends are minimal because of the Plexiglas walls acting as a thermal insulator.

Data are recorded using a National Instruments (NI) data acquisition system 9172. Due to the low voltage signal from T-type thermocouples, NI-DAQ module 9219 was used for all temperature measurements; this module was chosen as it provides simultaneous 24bit measurement which allows for highly accurate thermocouple measurement. Voltage measurements from the heat flux sensors were recorded using NI-DAQ module 9215 amplified by a factor of 1000 using Fylde amplifier 351UA; this setup allows for accurate time resolved measurements.

The heat flux sensor was calibrated by measuring the surface heat flux around the circumference of a single unconfined cylinder for a range of different Rayleigh numbers using a calibration technique described by Atmane et al. [4]. Using this method the heat flux sensor was rotated and measurements of the surface temperature and heat flux where recorded at $10^{\circ}$ intervals around the surface of the cylinder for varying Rayleigh numbers. An energy balance was then performed to determine a calibration factor to account for the difference between the power dissipated by the internal cartridge heaters measured using a Wattmeter and the factory calibration of the heat flux sensor. The mean Nusselt number of the cylinder was then calculated using the relationship shown in equation 3

$$
N \bar{u}=\frac{(\ddot{q})(D)}{(k)(\Delta T)}
$$




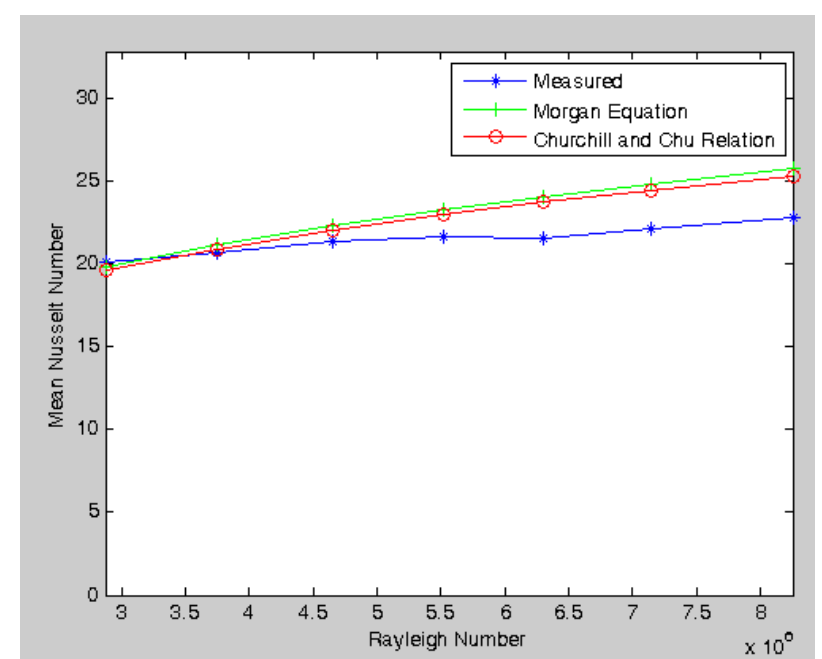

Figure 3. Heat Flux Sensor Calibration, Time Averaged Nusselt numbers against Rayleigh number, also showing correlations developed by Morgan [1] and Churchill and Chu [2]

During testing the first control parameter that was set was the spacing between the individual cylinders (S/D). Two separation distances were investigated in this study: $\mathrm{S}=2 \mathrm{D}$ and $\mathrm{S}=3 \mathrm{D}$. In addition to this, single cylinder tests were also investigated. The second control parameter of these experiments was the Rayleigh number. The Rayleigh number is the parameter that dictates the natural convection flow regime and which is given by the expression:

$$
R a=\frac{g \beta D^{3}\left(T_{s}-T_{\infty}\right) \operatorname{Pr}}{v^{2}}
$$

Where $\beta$ is the thermal expansion coefficient, $g$ is the gravitational constant, and $v$ is the kinematic viscosity of the fluid. All of the above parameters are evaluated at the film temperature which is defined as:

$T_{f}=\frac{T_{s}-T_{\infty}}{2}$

The Rayleigh number for a given test is therefore varied by adjusting the temperature difference $\Delta T$ between the cylinder temperature and the bulk water temperature. Tests were performed at Rayleigh numbers of $2 \times 10^{6}, 3 \times 10^{6}$ and $6 \times 10^{6}$.

Once the desired operating parameters were met the test section was rotated in $10^{\circ}$ intervals for half a revolution. At each interval the heat flux, surface temperature, and bulk fluid temperature were recorded. From initial preliminary testing it was found that for multiple cylinder testing, slow, very low frequency oscillations in heat transfer were apparent. Therefore in order to capture this oscillatory behaviour it was decided to test at each angle for a sampling time of approximately 220 s to ensure that all periodic behaviour was recorded. Samples were only recorded once a pseudo steady state was reached. Using the data recorded the local Nusselt number $(\mathrm{Nu})$ at each interval was calculated. In the work reported here the maximum Rayleigh number recorded was of the order of $10^{7}$, meaning that the flow is nominally still laminar.

\section{RESULTS AND DISCUSSION}

Results are presented for a single cylinder and a vertical inline cylinder pair with spacing of two and three cylinder diameters. The surface heat transfer is considered to be symmetrical about the vertical plane. As a result local variations are presented for half of the cylinder surface where $0^{\circ}$ represents the base of the cylinder and $180^{\circ}$ represents the top point of the cylinder. The time varying local Nusselt number is also presented with the spectral analysis result of the oscillating heat transfer.

\section{Single Cylinder}

Tests presented in figure 3 show the surface variation in the local Nusselt number of an unconfined single cylinder in water for angles between $0^{\circ}$ (bottom) and $180^{\circ}$ (top).

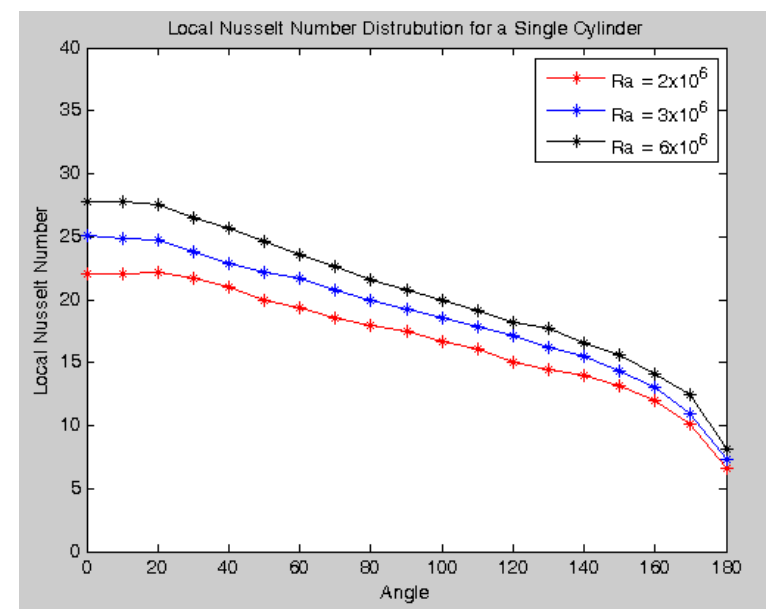

Figure 4. Local Nusselt number variation for a Single Cylinder

As can be clearly seen from figure 3, each of these results illustrate the same trend for each of the Rayleigh numbers employed. There is a gradual decrease in the local Nusselt number from $0^{\circ}$ to $160^{\circ}$ with an accelerated decrease occurring between $160^{\circ}$ and $180^{\circ}$. Research carried out by Saitoh et al. [11] has shown that this initial decrease in the surface heat transfer is a result of the thickening of the thermal boundary layer as the angle from the base of the cylinder is increased. From all single cylinder tests the maximum local Nusselt number occurs at the base of the cylinder; this maximum occurs as the boundary layer at this point is the thinnest resulting in the highest thermal gradient between the fluid in contact with the cylinder surface and the bulk fluid temperature. An accelerated decrease in the local Nusselt number occurs above 
an angle of $160^{\circ}$, this increased drop in the local Nusselt number has been proven to be caused [11] by detachment of the thermal plume from the cylinder surface. This greatly reduces the local thermal gradient and, as a result causes a drop off in the local Nusselt number. The mean Nusselt numbers for each of these single cylinder tests are listed in table 1 .

\section{Cylinder Pair: Time-averaged analysis}

Results are presented for a pair of vertically aligned cylinders at Rayleigh numbers of $2 \times 10^{6}, 3 \times 10^{6}$ and $6 \times 10^{6}$ and for vertical spacings of two and three cylinder diameters as presented in figure 4 to figure 6 .

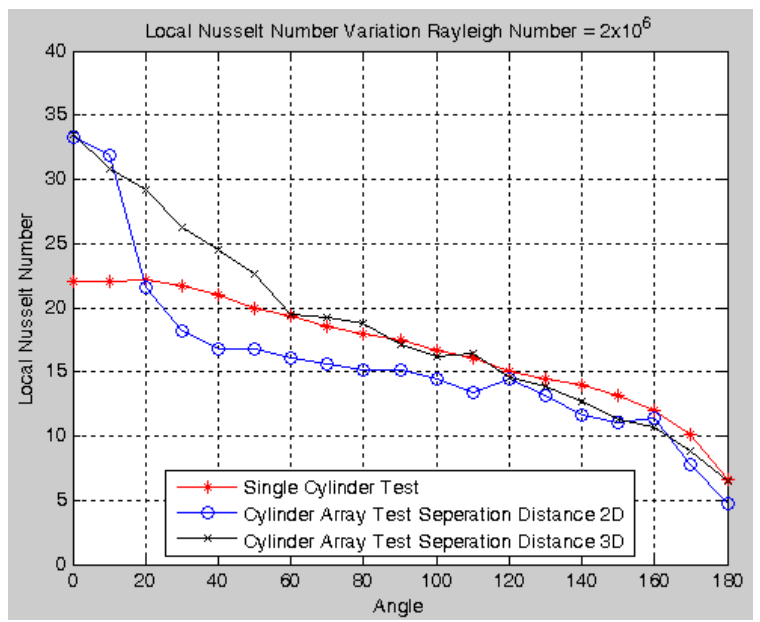

Figure 5. Local Nusselt Number Variation for a Rayleigh Number of $2 \times 10^{6}$

\begin{tabular}{|l|c|c|c|}
\hline $\begin{array}{l}\text { Approximate Average } \\
\text { Rayleigh Number }\end{array}$ & $\mathbf{2 x 1 0}^{\mathbf{6}}$ & $\mathbf{3 x 1 0}^{\mathbf{6}}$ & $\mathbf{6 x 1 0 ^ { \mathbf { 6 } }}$ \\
\hline $\begin{array}{l}\text { Mean Nusselt Number } \\
\text { Single Cylinder }\end{array}$ & 16.9 & 18.7 & 20.5 \\
\hline $\begin{array}{l}\text { Mean Nusselt Number } \\
\text { Vertical Spacing of 2D }\end{array}$ & 15.9 & 18.3 & 20.4 \\
\hline $\begin{array}{l}\text { Mean Nusselt Number } \\
\text { Vertical Spacing of 3D }\end{array}$ & 18.6 & 18.1 & 22.7 \\
\hline
\end{tabular}

Table 1. Mean Nusselt number Variation.

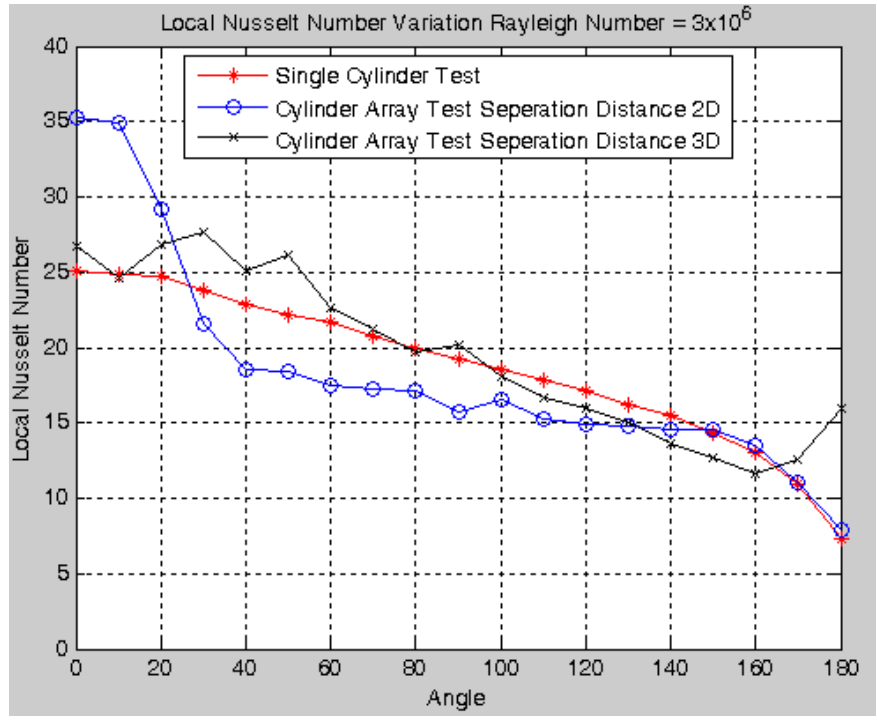

Figure 6. Local Nusselt Number Variation for a Rayleigh Number of $3 \times 10^{6}$

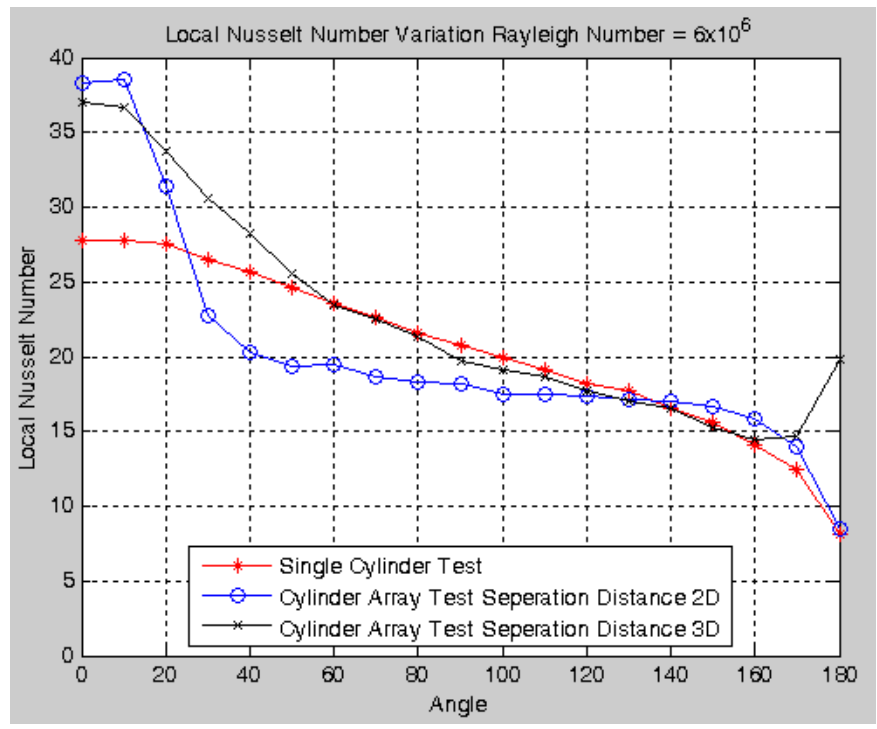

Figure 7. Local Nusselt Number Variation for a Rayleigh Number of $6 \times 10^{6}$

Figures 4-6 show that the local behaviour within an array differs greatly from that of a single cylinder. At both of the separation distances investigated the heat transfer at low angles far exceeds that of a single cylinder. Similar to the case of a single cylinder, the maximum local Nusselt number occurs close to the base of the cylinder. Maximum local enhancement occurred at a Rayleigh number of $2 \times 10^{6}$ with a cylinder spacing of 3D. For each test a sharper initial decrease in heat transfer was noted compared to that of a single cylinder. This dramatic difference would suggest that an additional mode of heat 
transfer is in effect at these initial angles rather than free convection alone, as occurs for a single cylinder.

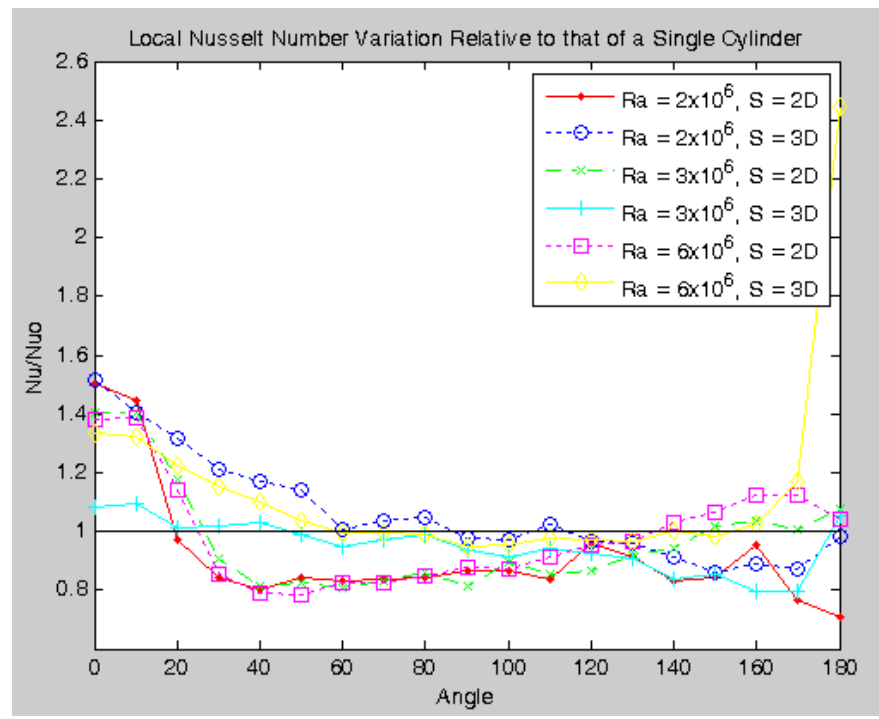

Figure 8. Local Nusselt number variation relative to that of a Single Cylinder

Figure 7 illustrates the local Nusselt number variation relative to that of a single cylinder. This figure highlights the fact that for a separation distance of $2 \mathrm{D}$ for the majority of the circumference of the upper cylinder the heat transfer is negatively affected by the presence of the lower cylinder. However, significant enhancement is evident at low angles less than $30^{\circ}$, possibly as a result of a forced convection effect from the lower cylinder. With a separation distance of 3D this initial enhancement region can be seen to extend over a larger envelope of the cylinder surface up to $60^{\circ}$. At this separation distance it can be seen that the heat transfer performance does not significantly drop below that of a single cylinder. Therefore overall an enhancement in heat transfer is noted at this separation distance within the investigated Rayleigh number range.

\section{Cylinder Pair: Time-resolved analysis}

Analysis of the heat transfer from the upper cylinder in a tubular array showed the presence of the lower cylinder causes oscillatory, periodic, heat transfer from the upper cylinder. This behaviour was found to be significantly dependent on the Rayleigh number of the fluids flow. This Rayleigh number dependence could suggest that this oscillatory behaviour is a result of vortex shedding from the upper cylinder due to the induced flow field from the lower cylinder. Figure 8 shows the time series and frequency plot of the local Nusselt number at a Rayleigh number of $2 \times 10^{6}$ and $6 \times 10^{6}$, a separation distance of $3 \mathrm{D}$ and an angular position of $0^{\circ}$. As can be seen from this figure at the higher Rayleigh number investigated the fluctuations occur less frequently but are of far greater magnitude.

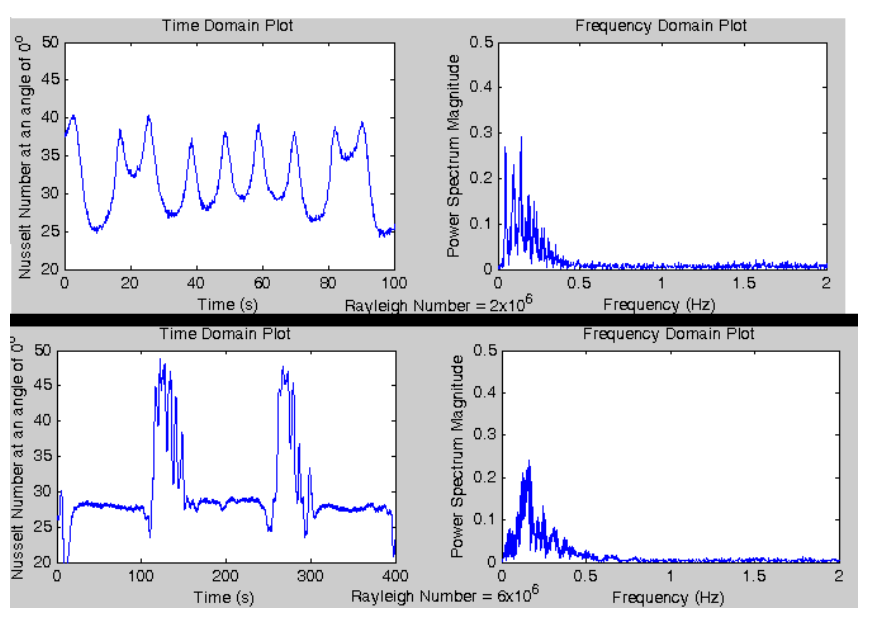

Figure 9. Variation in Nusselt number at $0^{\circ}$ on upper cylinder surface due to varying Rayleigh number, $S=3 D$.

For the Rayleigh number of $2 \times 10^{6}$ the dominant frequency of oscillation was found to occur at a frequency of $0.1417 \mathrm{~Hz}$ giving a period of oscillation of approximately $7 \mathrm{~s}$. For the higher Rayleigh number investigated oscillation was found to occur at a far lower frequency of $0.007 \mathrm{~Hz}$ giving a period of oscillation of approximately 143s. However as is evident in figure 8 these slow fluctuations dramatically affect the heat transfer from the upper cylinder. Such oscillatory behaviour is not present at this magnitude around a single cylinder. This behaviour could also be indentified as a possible mechanism of heat transfer enhancement due to increased mixing and fluid agitation around the upper cylinder. This theory is further supported by analysis of the local surface temperature variation under the previously specified conditions as presented in figure 9. As can be seen a periodic variation in the cylinder surface temperature is visible at the same periodic frequency as was noted for the local Nusselt number variation. The noted oscillation causes a decrease in the bar surface temperature. The authors suggest that this decrease in surface temperature could be a result of mixing caused by oscillation of the thermal plume drawing cold fluid from outside the thermal boundary layer to be drawn towards the cylinder surface, thus reducing the thickness of the thermal boundary layer and as a result increasing the surface heat transfer. This mixing effect due to plume oscillation could potentially be a further enhancement mechanism that occurs within an interacting cylinder array in addition to the enhancement mechanisms identified by Eckert and Soehngen [3]. 


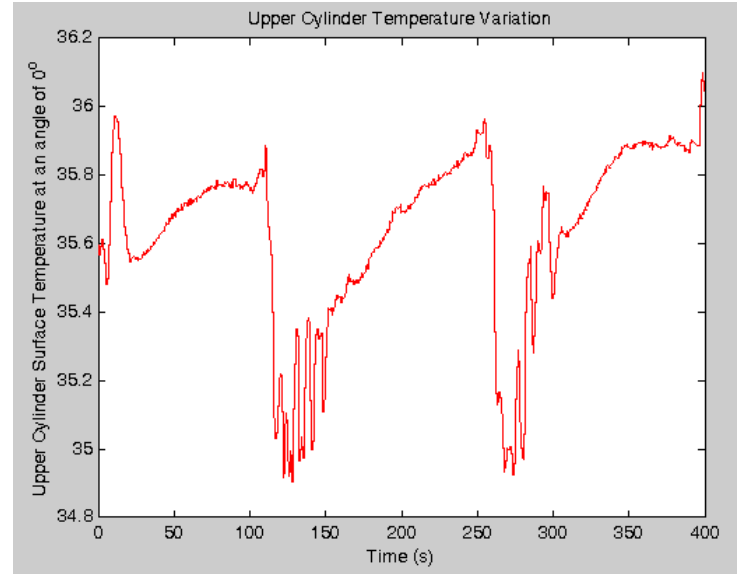

Figure 10. Surface Temperature variation of Upper cylinder at a Rayleigh number of $6 \times 10^{6}$ and a separation distance of 3D.

\section{CONCLUSIONS}

The local surface heat transfer distribution around a single cylinder and cylinders within an array were investigated for a separation distance of two and three cylinder diameters and for Rayleigh numbers of 2, 3 and $6 \times 10^{6}$. The fluctuating behaviour of the thermal plume within a cylinder array was analysed with a focus on the variation in the local Nusselt number from the surface of the upper cylinder as a result of this oscillation. Results were presented that suggest that oscillation of the thermal plume within a cylinder array induces mixing of the fluid surrounding the upper cylinder. This mixing may cause a reduction in the thickness of the thermal boundary layer and as a result enhancement of the associated heat transfer from the upper cylinder.

\section{NOMENCLATURE}

$\begin{array}{ll}N u & \text { Local Nusselt number } \\ R a & \text { Rayleigh number } \\ P r & \text { Prandtl number } \\ D & \text { Cylinder Diameter } \\ L & \text { Ceiling Height above Cylinder } \\ S & \text { Spacing between Cylinders } \\ g & \text { Gravitational constant } \\ B & \text { Thermal Expansion Co-efficient } \\ v & \text { Kinematic viscosity } \\ T_{f} & \text { Film Temperature } \\ T_{S} & \text { Cylinder Surface Temperature } \\ \mathrm{T} \infty & \text { Bulk Fluid Temperature } \\ \Delta T & \left(T_{s}-T_{\infty}\right) \\ N u o & \text { Local Nusselt number of a Single Cylinder } \\ k & \text { Thermal Conductivity of Water }\end{array}$

\section{ACKNOWLEDGMENTS}

The authors acknowledge the technical support staff of the Mechanical and Manufacturing Engineering Department of Trinity College Dublin. This research was funded by the Irish Research Council for Science Engineering and Technology.

\section{REFERENCES}

[1] Morgan, V. T., The Overall Convective Heat Transfer from a Smooth Circular Cylinder. Advance in Heat Transfer, Academic Press, New York, 1975. 11: p.199264.

[2] S.W. Churchill \& H.S. Chu, Correlating equations for laminar and turbulent free convection from a horizontal cylinder, 1974, Journal of heat and mass transfer Vol. 18 pp 1049 - 1053.

[3] Eckert, E. R. G., and Soehngen, E. E., Studies on Heat Transfer in Laminar Free Convection with ZedhnderMach Interferometer, Tech. Rept. No. 5747, U.S.A.F, Air Material Command, Dayton Ohio, 1948.

[4] M.A. Atmane, V.S. Chan, D.B. Murray, Natural Convection around a horizontal heated cylinder: The effects of Vertical Confinement, International Journal of Heat and Mass Transfer Vol. 46 No. 2 pp 3661 3672 .

[5] M.Yaghoubi, M. Ashjaee, A.H.Eshtiaghi, T.Yousefi, Thermal study of an array of inline horizontal cylinders below a nearly Adiabatic Ceiling, 2009, Experimental Thermal and Fluid Science, 33, pp $232-$ 239.

[6] I. Tokura, H. Saito, K. Kishinami, K. Muramoto, An Experimental Study of Free Convection Heat Transfer from a Horizontal Cylinder in a Vertical Array Set in Free Space Between Parallel Walls, 1983, Transactions of the ASME Vol. 105 pp $102-107$. G.Cesini, M. Paroncini, G. Cortella, M. Manzan, Natural Convection from a Horizontal Cylinder in a Rectangular Cavity, 1999, International Journal of Heat \& Mass Transfer Vol. 42 No. 10 pp1801 - 1811.

[8] E.M. Sparrow, J. E. Niethammer, Effect of Vertical Seperation Distance and Cylinder-to-Cylinder Temperature Imbalance on Natural Convection for a Pair of Horizontal Cylinders. 1981, Transactions of the ASME Vol. 103 pp 638- 644.

[9] T. Yousefi, M. Ashjaee. Experimental Study of Natural Convection heat transfer from vertical array of isothermal horizontal elliptic cylinders,2007, Experimental Thermal \& Fluid Science 32 pp240-48.

[10] Olivier Reymond, Darina B. Murray \& Tadhg S. O'Donovan, Natural Convection Heat Transfer from two Horizontal Cylinders, 2008, Experimental Thermal and Fluid Science 32 pp 1702 - 1709.

[11] T.Saitoh, T. Sajiki and K. Maruhara, Bench Mark Solutions to Natural Convection heat transfer problem around a horizontal Circular Cylinder, 1993, International Journal of Heat and Mass Transfer Vol. 36 No.5 pp $1251-125$ 
